\title{
Airway Smooth Muscle Hypercontractility in Asthma
}

\author{
Rachid Berair, Fay Hollins, and Christopher Brightling \\ Department of Infection, Inflammation and Immunity, Institute for Lung Health, University of Leicester, Leicester LE3 9QP, UK
}

Correspondence should be addressed to Christopher Brightling; ceb17@le.ac.uk

Received 24 July 2012; Accepted 28 January 2013

Academic Editor: Éric Rousseau

Copyright (C) 2013 Rachid Berair et al. This is an open access article distributed under the Creative Commons Attribution License, which permits unrestricted use, distribution, and reproduction in any medium, provided the original work is properly cited.

\begin{abstract}
In recent years, asthma has been defined primarily as an inflammatory disorder with emphasis on inflammation being the principle underlying pathophysiological characteristic driving airway obstruction and remodelling. Morphological abnormalities of asthmatic airway smooth muscle (ASM), the primary structure responsible for airway obstruction seen in asthma, have long been described, but surprisingly, until recently, relatively small number of studies investigated whether asthmatic ASM was also fundamentally different in its functional properties. Evidence from recent studies done on single ASM cells and on ASMimpregnated gel cultures have shown that asthmatic ASM is intrinsically hypercontractile. Several elements of the ASM contraction apparatus in asthmatics and in animal models of asthma have been found to be different from nonasthmatics. These differences include some regulatory contractile proteins and also some components of both the calcium-dependent and calcium-independent contraction signalling pathways. Furthermore, oxidative stress was also found to be heightened in asthmatic ASM and contributes to hypercontractility. Understanding the abnormalities and mechanisms driving asthmatic ASM hypercontractility provides a great potential for the development of new targeted drugs, other than the conventional current anti-inflammatory and bronchodilator therapies, to address the desperate unmet need especially in patients with severe and persistent asthma.
\end{abstract}

\section{Introduction}

Asthma is a chronic inflammatory disease characterized by variable airflow obstruction and bronchial hyperreactivity associated with airway remodelling [1]. Most of asthma symptoms result from airflow obstruction caused by airway lumen narrowing. Although this narrowing is multifactorial in origin, abnormalities of airway smooth muscle (ASM) structure and function have been identified as one of the main causes [2]. Increased ASM mass has long been recognized as a major component of airway remodelling [3,4]. More recently, asthmatic ASM was also found to be abnormal in its functional properties with increasing evidence showing intrinsic heightened contractility independent of other structural cells and independent of the asthma inflammatory milieu. In this paper we will examine the evidence of ASM hypercontractility in asthmatics, explore the potential mechanisms driving it, discuss its relevance, and briefly suggest its role in future asthma therapy.

\section{Evidence of ASM Hypercontractility in Asthmatics}

Abnormalities of asthmatic ASM structure and morphology have been described by Huber and Koesser more than 90 years ago when they reported increased ASM mass in a small group of patients who died of status asthmaticus compared to ASM form patients who died from nonpulmonary conditions [3]. This structural association has since been extensively described [4] although whether asthmatic ASM is also abnormal in function and if so whether this abnormality is an inherent property or only a result of the asthma inflammatory milieu has long been an unresolved question [5]. A few in vitro studies from the 1980-90s have tried to address this issue but the results have largely been conflicting. Compared to nonasthmatic controls, some studies suggested increased force generation in asthmatics ASM preparations; others showed no difference and even some seem to suggest decreased force generation in asthmatics [6-13]. Most of these studies had major methodological and statistical limitations 
such as small sample size, failure to measure force per crosssectional area (stress), failure to measure ASM shortening, and failure to identify ideal lengths for maximal contraction [14]. Furthermore, none of these studies examined ASM contractility at a cellular level, thus the mechanical effect of the extracellular nonmuscular connective tissue, and the biological effect of inflammatory cells and cytokines present in ASM preparations, on the final results could not be determined.

The first robust evidence of ASM hypercontractility in asthmatics was reported by $\mathrm{Ma}$ et al. This was the first study attempting to assess contractility characteristics of asthmatic ASM at cell level [15]. The study included 5 asthmatics and a similar number of nonasthmatic controls. 10-20 ASM cells were isolated from endobronchial biopsies collected from each subject. Maximum capacity and velocity of shortening of zero loaded single ASM cells in response to electrical field stimulation were measured under inverted phase-contrast microscopy. Asthmatics ASM cells showed significantly increased maximum capacity and velocity of shortening compared to controls. Although in this study the maximum shortening capacity in asthmatic ASM cells was increased by almost a third compared to controls, it should be considered that this shortening was measured at zero load and ASM cells in vivo would very likely shorten by a much lesser degree. This observation is pivotal but needs to be interpreted with caution due to the small sample size of this study.

Matsumoto et al. assessed asthmatic ASM contractility using a collagen gel contraction assay [16]. Gel percentage contraction to histamine was measured using floating gels containing ASM from 8 subjects with asthma and 9 nonasthmatic controls. These ASM containing gels were incubated overnight using 2 methods: attached or unattached to casting plates. The study found, using both methods, that histamineexposed gels containing asthmatic ASM contracted more significantly.

More recently, Sutcliffe et al. also used gel contraction assay to assess ASM contractility in a much larger sample of 19 asthmatics and 8 healthy controls [17]. Gel contraction was measured every 15 minutes after stimulation with bradykinin. Results again showed significantly increased agonist-induced contraction in the asthma group (Figure 1).

Importantly, phenotypic plasticity of structural cells in culture cannot be completely excluded from studies done on primary ASM cultures, but we think this, if present, was minimal. The above evidence, in our opinion, confirms that asthmatic ASM is fundamentally different and hypercontractile and that this hypercontractility is a basic property and is independent from other asthma structural cells and airway inflammation, although in vivo these may play an important role in modulating the hypercontractile response.

\section{Potential Mechanisms Driving ASM Hypercontractility in Asthmatics}

3.1. Physiology of Human ASM Contraction. As in all muscle cells, contraction in ASM is initiated by increased cytosolic calcium ions $\left(\mathrm{Ca}^{+2}\right)$ level, though, unlike most other muscle cells, the source of this $\mathrm{Ca}^{2+}$ surge in ASM is mainly from intracellular sarcoplasmic reticulum (SR) stores rather than from the usual extracellular $\mathrm{Ca}^{2+}$ influx through voltagedependent calcium channels during depolarization seen in cardiac, skeletal, and vascular muscle cells.

The sequence of events leading to the contraction of an ASM cell starts with the interaction of a contractile agonist with its G-protein-coupled receptors (Figure 2). This results in the activation of phospholipase C (PLC), which in turn leads to the formation of the inositol triphosphate $\left(\mathrm{IP}_{3}\right)$ through the hydrolyzation of phosphatidylinositol bisphosphate $\left(\mathrm{PIP}_{2}\right) . \mathrm{IP}_{3}$ then binds to its receptor on SR membrane releasing $\mathrm{Ca}^{2+}$ stores which then, through forming a complex with calmodulin, activate myosin light chain kinase (MLCK) which phosphorylates regulatory myosin light chains (rMLC) forming p-MLC [18]. Finally, this leads to the activation of actin and myosin crossbridges resulting in shortening and contraction [19]. After initiation of contraction, cytosolic $\mathrm{Ca}^{2+}$ levels return to normal through different mechanisms including pumping out of the cell by the plasma membrane $\mathrm{Ca}^{2+}$-ATPase (PMCA) and the sodium calcium exchanger (NCX), binding to cytosolic proteins, uptake by mitochondria, and also reuptake to the SR through the action of the sarco/endoplasmic reticulum $\mathrm{Ca}^{2+}$ ATPase (SERCA) [20].

Another mechanism for agonist-induced $\mathrm{Ca}^{2+}$ released from SR is through ryanodine receptors (RyR). This is mediated by membrane CD38 and nucleotide metabolite cyclic ADP-ribose (cADPR) [21]. The RyR channels are also activated through localized elevation of $\mathrm{Ca}^{2+}$ levels $\left(\mathrm{Ca}^{2+}\right.$ induced $\mathrm{Ca}^{2+}$ release).

The phosphorylation of rMLC is also regulated by myosin light chain phosphatase (MLCP) which converts p-MLC back to inactive rMLC. MLCP activity is modulated through two agonist-induced mechanisms in a process called calcium sensitization. First, it is controlled by the inhibitory action of diacylglycerol (DAG), another second messenger, which also results from the hydrolyzation of $\mathrm{PIP}_{2}$. DAG activates Protein Kinase C (PKC) which in turn inhibits MLCP through phosphorylation. Second, MLCP is also negatively controlled by RhoA and its target Rho Kinase, which deactivates MLCP similarly through phosphorylation.

Exploring the possible mechanisms of asthmatic ASM hypercontractility is a difficult task as the evidence is less well established with relatively few human studies. Hypercontraction of asthmatic ASM could be due to abnormalities in one or more of these components or steps of ASM contraction model. The complexity of investigating differences in signalling or contractile proteins is that the abnormality could be in a number of levels. This could be at gene, gene expression (epigenetics), or, more commonly, at protein phosphorylation level.

3.2. Abnormalities of Contractile Proteins. The most characterized potentially abnormal component of the contraction apparatus in asthmatic ASM is MLCK, a key regulator of ASM contraction. Increased MLCK levels have been reported in sensitized animal and human airways [22, 23]. As part 


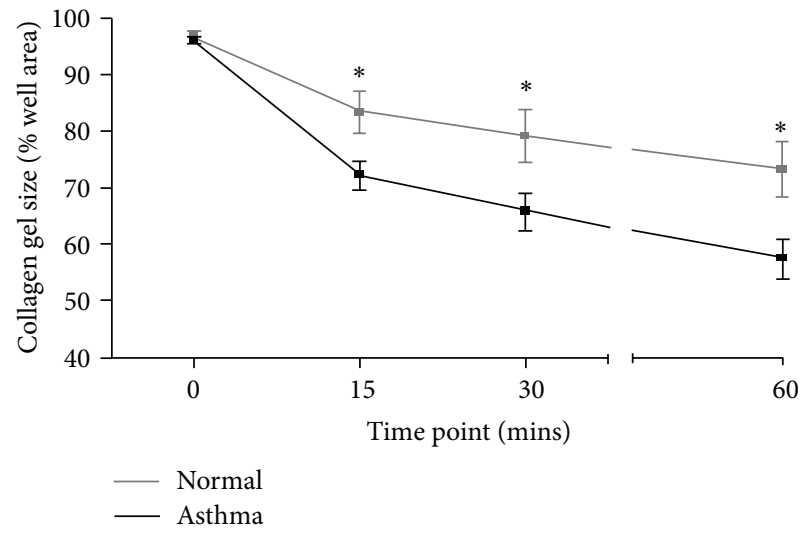

(a)

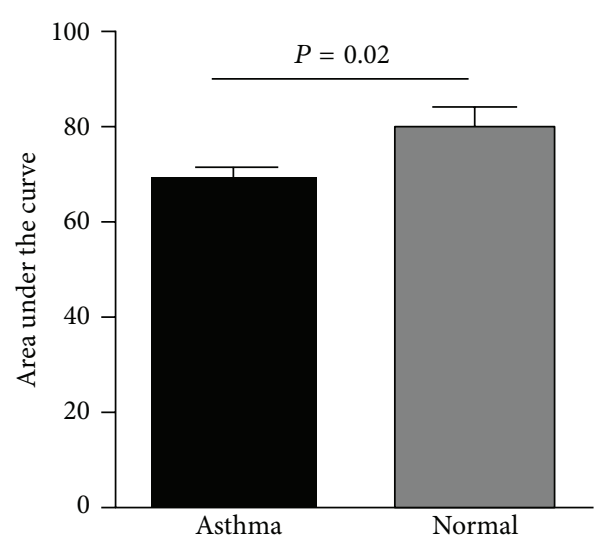

(b)

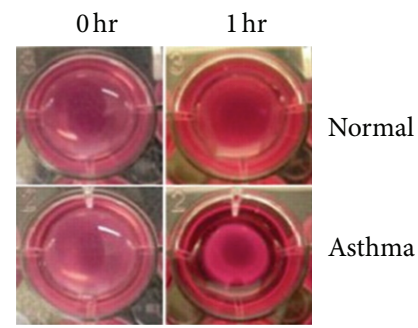

(c)

Figure 1: (a) Percentage contraction of collagen gels impregnated with airway smooth muscle from donors with asthma $(n=19)$ versus healthy control donors ( $n=8$ ) over 1 hour following stimulation with $1 \mathrm{~nm}$ bradykinin, (b) area under the curve gel contraction (mean [SEM]), and (c) representative gel photographs taken at 0 hour and 1 hour time points. The comparison was made by unpaired $t$-test. ${ }^{*} P<0.05$. [17]. (Reprinted with permission of the American Thoracic Society. Copyright (C) 2012 American Thoracic Society. Amanda Sutcliffe, Fay Hollins, Edith Gomez, Ruth Saunders, Camille Doe, Marcus Cooke, R. A. John Challiss, and Chris E. Brightling/2012/ Increased Nicotinamide Adenine Dinucleotide Phosphate Oxidase 4 Expression Mediates Intrinsic Airway Smooth Muscle Hypercontractility in Asthma. American Journal of Respiratory and Critical Care Medicine/Vol. 185/pp 267-274. (An official Journal of The American Thoracic Society).

of the same contractility study described earlier, Ma et al. assessed MLCK expression by using RT-PCR to measure mRNA. Measuring MLCK protein was not possible due to the small cell sample (10-20 cells per subject) [15]. MLCK mRNA was significantly increased in asthmatics compared to a group of both allergic and nonallergic nonasthmatic controls.

Benayoun et al. examined contractile protein expression in biopsies in asthmatics with different asthma severity compared to nonasthmatic controls and also compared to patients with COPD [24]. Although $\alpha$-actin and myosin heavy chain isoforms (SM1, SM2) expression was similar in all groups, MLCK expression was increased in all patients with asthma and COPD compared to controls. Furthermore, MLCK expression was significantly more in patients with severe asthma compared to all other groups. Interestingly, although p-MLC, the active product from the action of MLCK, was detected only in the asthmatic groups, this was not statistically significant.

Not all studies showed increased MLCK expression or content in asthmatics. Matsumoto et al. demonstrated no increase in MLCK content in cultured asthmatic ASM. The authors did admit that this negative result could be due to possible degradation of MLCK during the harvesting stage of ASM cells [16]. Moreover, Woodruff et al. showed no increased gene expression of any of the contractile proteins MLCK, MCH, SM22, or $\alpha$-actin in a sample of 11 asthmatics compared to 8 controls, although this could be due to the fact that the asthmatics in this study had only mild disease [25].

In vivo, the degree of mast cell infiltration of ASM, a histopathological feature of asthma [26, 27], has been shown to be positively associated with increased $\alpha$-actin expression [28]. Moreover, in vitro coculture of human ASM with human lung mast cell (HLMC), or $\beta$-tryptase, a serine protease released by mast cells following activation, resulted in increased $\alpha$-actin expression and increased ASM contraction. This has been shown to be mediated through autocrine upregulation of transforming growth factor $\beta 1$ (TGF- $\beta 1$ ) in ASM [28]. Histamine release from mast cells in a piece-meal fashion as demonstrated by mast cells within the ASM bundle in vivo by electron microscopy [27] and in vitro 


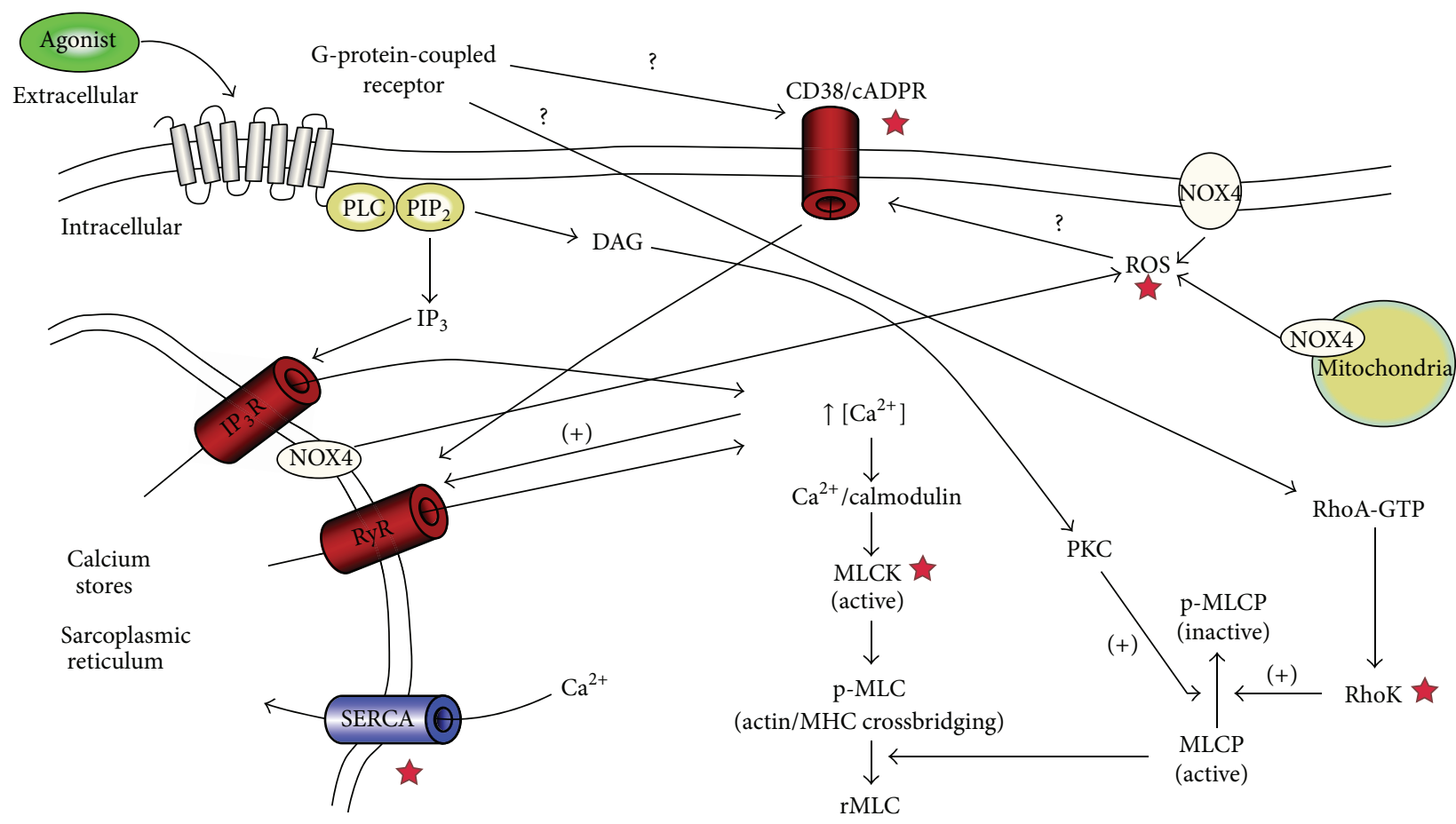

FIGURE 2: Overview of the signalling pathways involved in airway smooth muscle contraction. The contractile agonist interacts with its specific G-protein-coupled receptor (GPCR) leading to the activation of phospholipase C (PLC) which hydrolyzes phosphatidylinositol bisphosphate $\left(\mathrm{PIP}_{2}\right)$ leading to the formation of two-second messengers, inositol triphosphate $\left(\mathrm{IP}_{3}\right)$, and diacylglycerol (DAG). IP 3 interacts with its receptor $\left(\mathrm{IP}_{3} \mathrm{R}\right)$ on the sarcoplasmic reticulum (SR) leading to the release of calcium ions $\mathrm{Ca}^{2+}$ which in turn, through forming a complex with calmodulin, activates myosin light chain kinase (MLCK). MLCK phosphorylates regulatory myosin light chain (rMLC) to form p-MLC which leads to myosin and actin crossbridging and contraction. p-MLC is deactivated by the action of myosin light chain phosphatase (MLCP). Both DAG, through its action on protein kinase C (PKC), and RhoA, through its target Rho Kinase (RhoK), have an inhibitory action on MLCP through phosphorylation. Interaction of agonist with GPCR also activates both CD38/cADPR and Rho/RhoK pathways, although the exact mechanism is not fully known. CD38/cADPR activation leads to the release of $\mathrm{Ca}^{2+}$ from SR through ryanodine receptors (RyR) channels. Nicotinamide adenine dinucleotide phosphate oxidase type 4 (NOX4) generates reactive oxygen species (ROS) which may affect ASM calcium homeostasis and subsequently ASM contraction through their action on the CD38/cADPR pathway. Red stars $\star$ indicate signalling points with abnormalities suspected of driving hypercontractility in asthmatic ASM.

following fibroblastoid differentiation [29, 30] might exert a direct spasmogen effect upon the ASM in asthma. Mast cells are also an important source of cytokines including the Th2 cytokine IL-13 [31]. Mast cells localized in the ASMbundle express IL-13, particularly in severe disease which can prime ASM to a more hyper-contractile state [31-33]. Abnormalities of $\alpha$-actin expression in asthmatic ASM have only been described in this context and previous studies examining $\alpha$ actin expression in asthmatics were mostly negative $[24,34]$.

\subsection{Dysregulation of Calcium Homeostasis. As discussed} earlier, calcium plays a central role in ASM contraction. There is an increasing pool of evidence showing abnormal calcium homeostasis in asthmatic ASM, thus, suggesting that abnormal calcium handling, signaling, or storage as possible underlying mechanisms for asthmatic ASM hypercontractility is a plausible argument.

In general, factors leading to increased cytosolic $\mathrm{Ca}^{2+}$ levels result in increased ASM contraction. Mahn et al. examined SERCA expression in ASM from asthmatics and healthy volunteers [35]. The expression of SERCA2 isoform mRNA, the main isoform expressed in human ASM, was reduced in patients with moderately severe asthma. This was found in in vivo samples and also on ASM cultures. Reduction of SERCA2 expression in healthy control ASM culture by siRNA resulted in phenotypic shifting to an asthmatic ASM type with increased motility, secretion, and slow $\mathrm{Ca}^{2+}$ recovery. Of interest, $\mathrm{IP}_{3} \mathrm{R}$ mRNA expression was not increased in the asthmatics group.

Another signalling pathway that has the potential of altering calcium homeostasis and increasing contractility is the CD38/cADPR/RyR pathway. CD38 deficiency in animals has been shown to inhibit airway hyperresponsiveness (AHR) [36]. In human ASM, TNF- $\alpha$, IL- $1 \beta$, IL-13, and IFN- $\gamma$ were all found to increase CD38 expression, cADPR activity, and $\mathrm{Ca}^{2+}$ response to various natural contractile agonists $[37,38]$. Moreover, in another study, highlighting the possibility that CD38 abnormalities could be a fundamental characteristic in asthma, TNF- $\alpha$ was shown to significantly increase CD38 expression in asthmatic ASM than in controls [39]. 
Altered calcium homeostasis in asthmatic ASM is also due to altered extracellular calcium influx through nonvoltage-dependent channels [40]. Although this has been directly implicated in altered mitochondrial biogenesis and increased ASM proliferation in asthmatics, its relevance to hypercontractility remains to be investigated.

3.4. Abnormal Calcium Sensitization. Upregulation of the calcium independent RhoA/Rho Kinase signalling pathway leading to inhibition of MLCP would result in increased levels of p-MLC and subsequently increased ASM contraction force at the same $\mathrm{Ca}^{2+}$ concentration. Abnormalities of this signalling pathway have been described in animal models of various smooth muscle disorders including hypertension, coronary artery spasm, and preterm labour [41]. This has also been described in animal models of allergic bronchial asthma [42]. Increased levels of RhoA protein and RhoA mRNA were found in airway hyperresponsive rat models although this is probably medicated through inflammatory cytokines [41-43].

We emphasize that most of the abnormalities of calcium homeostasis and calcium sensitization explored in this paper were only described in single studies which have not been replicated; thus their importance remains to be fully determined.

3.5. Increased Oxidative Stress Burden. Although reactive oxygen species (ROS) play an important physiological role in different cellular functions, excessive production results in the tissue damage seen in a range of chronic and acute diseases. Oxidative stress burden is increased in bronchial asthma with recent evidence identifying an increase in the generation of ROS in asthmatic ASM in vivo and in primary ASM cultures [17]. This was inversely correlated to the degree of airflow obstruction and AHR. More importantly, nicotinamide adenine dinucleotide phosphate oxidase type 4 (NOX4) expression, an important source of ROS, was increased in asthmatics ASM. Moreover, increased asthmatics ASM contractility seen in gel contraction essay was abolished by adding NOX4 inhibiters or NOX4 small interfering RNA.

3.6. SMAD3 and ORMDL3. Genome-wide association studies (GWASs) have identified several associations between multiple single-nucleotide polymorphisms (SNPs) on a number of locations and asthma. One such SNP is on the SMAD3 gene. SMAD3 gene, located on chromosome 15, encodes for a similarly named protein, SMAD3 protein. This protein is a signal transduction and transcription modulator and is activated by TGF- $\beta$ which has a complex role in cell growth and proliferation and is involved in airway inflammation and remodelling in asthma. As mentioned earlier, upregulation of TGF- $\beta 1$ observed on coculture of ASM and HLMC was associated with increased $\alpha$-actin expression and increased contractility of ASM [28].

Another association identified by GWASs was on the ORMDL3 gene $[44,45]$. This gene encodes for the SR membrane protein ORMDL3 which is thought to have an important role in calcium homeostasis possibly through its action on SERCA. Overexpression of ORMDL3 was found to be associated with reduced SERCA activity as evidenced by higher basal cytosolic $\mathrm{Ca}^{2+}$ levels, lower $\mathrm{SR} \mathrm{Ca}^{2+}$ levels, and slower $\mathrm{Ca}^{2+}$ reuptake into the SR [46]. Thus, based on the above evidence, polymorphism of SMAD3 or ORMDL3 could be implicated in the hypercontractility seen in asthmatic ASM through their effect on $\alpha$-actin expression and calcium homeostasis, respectively.

\section{Clinical Relevance of Asthmatic ASM Hypercontraction}

The contraction of ASM in asthma causes airway obstruction [47]. Although we know that AHR is predominantly a function of ASM, how much of it is driven by ASM hypercontractility is a difficult and controversial question to answer. We do recognize that some of ASM hypercontractility is contributed to by airway inflammation and inflammatory cytokines, but there is also good evidence to suggest that there is more to AHR than inflammation. Although corticosteroids reduce AHR, studies using agents that target specific parts of inflammation, in the form of antibodies against IL-5 and IgE, significantly improved inflammation but did not affect AHR [48-50].

Therapies that reduce ASM contraction have been shown to improve asthma symptoms, lung function, and maybe even AHR. The roles of $\beta 2$-agonists and anticholinergics in the treatment of asthma are well established. Another evidence of the significance of ASM is from bronchial thermoplasty (BT). $\mathrm{BT}$, where radiofrequency energy is used on airways to reduce ASM mass, was shown to improve asthma control, quality of life, and, in one study, AHR [51]. Further studies are required to examine whether the efficacy of BT in asthmatics is related to changes in ASM mass and how this relates to changes in airway structure, physiology, and clinical expression of disease.

\section{ASM Hypercontractility and the Future of Asthma Therapy}

The desperate need for new asthma treatments is universally acknowledged. Asthma incidence is increasing with more than half of the patients failing to achieve adequate control. Furthermore, 5-10\% of patients have persistent symptoms despite maximal treatment with conventional antiinflammatory and bronchodilator therapy $[1,52]$.

Detailed discussion of the future of asthma treatment is beyond the scope of this paper. Targeted drugs that would act on specific aspects of inflammation and contractility are the way forward. Over the last few years, a huge research effort has been on trying to identify asthma phenotypes based on inflammation, but much less was dedicated to addressing contractility. Several chemicals have been found to reduce ASM gel contraction in vitro including inhibitors of phospholipase C, myosin light chain kinase, Rho kinase, and NOX4 and thus this has identified these enzymes as potential targets for future novel asthma treatments $[16,17]$. 


\section{Conclusion}

In conclusion, we believe, based on the evidence reviewed, that ASM in asthmatics is hypercontractile. Airway inflammation contributes to and augments ASM hypercontractility but is neither sufficient nor necessary. Indeed evidence presented here suggests that ASM hypercontractility is an intrinsic abnormality in asthma that persists in primary culture in the absence of the asthmatic environment. Improved understanding of the mechanisms driving this hypercontractility will pave the way for future treatments that will address contractility, achieve better relief of airway obstruction, and impact on asthma control and exacerbations.

\section{Acknowledgment}

C. Brightling is funded by a Wellcome Senior Clinical Fellowship.

\section{References}

[1] E. D. Bateman, S. S. Hurd, P. J. Barnes et al., "Global strategy for asthma management and prevention: GINA executive summary," European Respiratory Journal, vol. 31, no. 1, pp. 143-178, 2008.

[2] J. G. Martin, A. Duguet, and D. H. Eidelman, “The contribution of airway smooth muscle to airway narrowing and airway hyperresponsiveness in disease," European Respiratory Journal, vol. 16, no. 2, pp. 349-354, 2000.

[3] H. Huber and K. Koesser, "pathology of bronchial asthma," Archives of Internal Medicine, pp. 689-760, 1922.

[4] A. James and N. Carroll, "Airway smooth muscle in health and disease; methods of measurement and relation to function," European Respiratory Journal, vol. 15, no. 4, pp. 782-789, 2000.

[5] R. A. Panettieri Jr., M. I. Kotlikoff, W. T. Gerthoffer et al., "Airway smooth muscle in bronchial tone, inflammation, and remodeling: basic knowledge to clinical relevance," American Journal of Respiratory and Critical Care Medicine, vol. 177, no. 3, pp. 248-252, 2008.

[6] A. M. Bramley, R. J. Thomson, C. R. Roberts, and R. R. Schellenberg, "Hypothesis: excessive bronchoconstriction in asthma is due to decreased airway elastance," European Respiratory Journal, vol. 7, no. 2, pp. 337-341, 1994.

[7] T. R. Bai, "Abnormalities in airway smooth muscle in fatal asthma: a comparison between trachea and bronchus," American Review of Respiratory Disease, vol. 143, no. 2, pp. 441-443, 1991.

[8] J. C. de Jongste, H. Mons, I. L. Bonta, and K. F. Kerrebijn, "In vitro responses of airways from an asthmatic patient," European Journal of Respiratory Diseases, vol. 71, no. 1, pp. 23-29, 1987.

[9] T. Bjorck, L. E. Gustafsson, and S. E. Dahlen, "Isolated bronchi from asthmatics are hyperresponsive to adenosine, which apparently acts indirectly by liberation of leukotrienes and histamine," American Review of Respiratory Disease, vol. 145, no. 5, pp. 1087-1091, 1992.

[10] R. G. Goldie, D. Spina, and P. J. Henry, "In vitro responsiveness of human asthmatic bronchus to carbachol, histamine, $\beta$ adrenoceptor agonists and theophylline," British Journal of Clinical Pharmacology, vol. 22, no. 6, pp. 669-676, 1986.

[11] J. A. Roberts, D. Raeburn, I. W. Rodger, and N. C. Thomson, "Comparison of in vivo airway responsiveness and in vitro smooth muscle sensitivity to methacholine in man," Thorax, vol. 39, no. 11, pp. 837-843, 1984.

[12] S. D. Whicker, C. L. Armour, and J. L. Black, "Responsiveness of bronchial smooth muscle from asthmatic patients to relaxant and contractile agonists," Pulmonary Pharmacology, vol. 1, no. 1, pp. 25-31, 1988.

[13] J. Cerrina, C. Labat, I. Haye-Legrande, B. Raffestin, J. Benveniste, and C. Brink, "Human isolated bronchial muscle preparations from asthmatic patients: effects of indomethacin and contractile agonists," Prostaglandins, vol. 37, no. 4, pp. 457469, 1989.

[14] C. Y. Seow, R. R. Schellenberg, and P. D. Pare, "Structural and functional changes in the airway smooth muscle of asthmatic subjects," American Journal of Respiratory and Critical Care Medicine, vol. 158, no. 5, pp. S179-S186, 1998.

[15] X. Ma, Z. Cheng, H. Kong et al., "Changes in biophysical and biochemical properties of single bronchial smooth muscle cells from asthmatic subjects," American Journal of Physiology, vol. 283, no. 6, pp. L1181-L1189, 2002.

[16] H. Matsumoto, L. M. Moir, B. G. G. Oliver et al., "Comparison of gel contraction mediated by airway smooth muscle cells from patients with and without asthma," Thorax, vol. 62, no. 10, pp. 848-854, 2007.

[17] A. Sutcliffe, F. Hollins, E. Gomez et al., "Increased nicotinamide adenine dinucleotide phosphate oxidase 4 expression mediates intrinsic airway smooth muscle hypercontractility in asthma," American Journal of Respiratory and Critical Care Medicine, vol. 185, pp. 267-274, 2012.

[18] M. J. Berridge, "Inositol trisphosphate and calcium signalling mechanisms," Biochimica et Biophysica Acta, vol. 1793, no. 6, pp. 933-940, 2009.

[19] S. J. Gunst and D. D. Tang, "The contractile apparatus and mechanical properties of airway smooth muscle," European Respiratory Journal, vol. 15, no. 3, pp. 600-616, 2000.

[20] E. Roux and M. Marhl, "Role of sarcoplasmic reticulum and mitochondria in $\mathrm{Ca}^{2+}$ removal in airway myocytes," Biophysical Journal, vol. 86, no. 4, pp. 2583-2595, 2004.

[21] D. A. Deshpande, T. A. White, S. Dogan, T. F. Walseth, R. A. Panettieri, and M. S. Kannan, "CD38/cyclic ADP-ribose signaling: role in the regulation of calcium homeostasis in airway smooth muscle," American Journal of Physiology, vol. 288, no. 5, pp. L773-L788, 2005.

[22] A. J. Ammit, C. L. Armour, and J. L. Black, "Smooth-muscle myosin light-chain kinase content is increased in human sensitized airways," American Journal of Respiratory and Critical Care Medicine, vol. 161, no. 1, pp. 257-263, 2000.

[23] S. K. Kong, A. J. Halayko, and N. L. Stephens, "Increased myosin phosphorylation in sensitized canine tracheal smooth muscle," American Journal of Physiology, vol. 259, no. 2, pp. L53-L56, 1990.

[24] L. Benayoun, A. Druilhe, M. C. Dombret, M. Aubier, and M. Pretolani, "Airway structural alterations selectively associated with severe asthma," American Journal of Respiratory and Critical Care Medicine, vol. 167, no. 10, pp. 1360-1368, 2003.

[25] P. G. Woodruff, G. M. Dolganov, R. E. Ferrando et al., "Hyperplasia of smooth muscle in mild to moderate asthma without changes in cell size or gene expression," American Journal of Respiratory and Critical Care Medicine, vol. 169, no. 9, pp. 10011006, 2004.

[26] C. E. Brightling, P. Bradding, F. A. Symon, S. T. Holgate, A. J. Wardlaw, and I. D. Pavord, "Mast-cell infiltration of 
airway smooth muscle in asthma," The New England Journal of Medicine, vol. 346, no. 22, pp. 1699-1705, 2002.

[27] H. Begueret, P. Berger, J. M. Vernejoux, L. Dubuisson, R. Marthan, and J. M. Tunon-de-Lara, "Inflammation of bronchial smooth muscle in allergic asthma," Thorax, vol. 62, no. 1, pp. 8$15,2007$.

[28] L. Woodman, S. Siddiqui, G. Cruse et al., "Mast cells promote airway smooth muscle cell differentiation via autocrine upregulation of TGF- $\beta 1$," Journal of Immunology, vol. 181, no. 7, pp. 5001-5007, 2008.

[29] F. Hollins, D. Kaur, W. Yang et al., "Human airway smooth muscle promotes human lung mast cell survival, proliferation, and constitutive activation: cooperative roles for CADM1, stem cell factor, and IL-6," Journal of Immunology, vol. 181, no. 4, pp. 2772-2780, 2008.

[30] D. Kaur, R. Saunders, F. Hollins et al., "Mast cell fibroblastoid differentiation mediated by airway smooth muscle in asthma," Journal of Immunology, vol. 185, no. 10, pp. 6105-6114, 2010.

[31] C. E. Brightling, F. A. Symon, S. T. Holgate, A. J. Wardlaw, I. D. Pavord, and P. Bradding, "Interleukin-4 and - 13 expression is co-localized to mast cells within the airway smooth muscle in asthma," Clinical and Experimental Allergy, vol. 33, no. 12, pp. 1711-1716, 2003.

[32] J. C. Laporte, P. E. Moore, S. Baraldo et al., "Direct effects of interleukin-13 on signaling pathways for physiological responses in cultured human airway smooth muscle cells," American Journal of Respiratory and Critical Care Medicine, vol. 164, no. 1, pp. 141-148, 2001.

[33] S. K. Saha, M. A. Berry, D. Parker et al., "Increased sputum and bronchial biopsy IL-13 expression in severe asthma," Journal of Allergy and Clinical Immunology, vol. 121, no. 3, pp. 685-691, 2008.

[34] R. Léguillette, M. Laviolette, C. Bergeron et al., "Myosin, transgelin, and myosin light chain kinase expression and function in asthma," American Journal of Respiratory and Critical Care Medicine, vol. 179, no. 3, pp. 194-204, 2009.

[35] K. Mahn, S. J. Hirst, S. Ying et al., "Diminished sarco/endoplasmic reticulum $\mathrm{Ca}^{2+}$ ATPase (SERCA) expression contributes to airway remodelling in bronchial asthma," Proceedings of the National Academy of Sciences of the United States of America, vol. 106, no. 26, pp. 10775-10780, 2009.

[36] A. G. P. Guedes, J. Paulin, L. Rivero-Nava, H. Kita, F. E. Lund, and M. S. Kannan, "CD38-deficient mice have reduced airway hyperresponsiveness following IL-13 challenge," American Journal of Physiology, vol. 291, no. 6, pp. L1286-L1293, 2006.

[37] D. A. Deshpande, T. F. Walseth, R. A. Panettieri, and M. S. Kannan, "CD38/cyclic ADP-ribose-mediated $\mathrm{Ca}^{2+}$ signaling contributes to airway smooth muscle hyper-responsiveness," The FASEB Journal, vol. 17, no. 3, pp. 452-454, 2003.

[38] D. A. Deshpande, S. Dogan, T. F. Walseth et al., "Modulation of calcium signaling by interleukin-13 in human airway smooth muscle: role of $\mathrm{CD} 38 /$ cyclic adenosine diphosphate ribose pathway," American Journal of Respiratory Cell and Molecular Biology, vol. 31, no. 1, pp. 36-42, 2004.

[39] J. A. Jude, M. E. Wylam, T. F. Walseth, and M. S. Kannan, "Calcium signaling in airway smooth muscle," Proceedings of the American Thoracic Society, vol. 5, no. 1, pp. 15-22, 2008.

[40] T. Trian, G. Benard, H. Begueret et al., "Bronchial smooth muscle remodeling involves calcium-dependent enhanced mitochondrial biogenesis in asthma," Journal of Experimental Medicine, vol. 204, no. 13, pp. 3173-3181, 2007.
[41] Y. Chiba, K. Matsusue, and M. Misawa, "RhoA, a possible target for treatment of airway hyperresponsiveness in bronchial asthma," Journal of Pharmacological Sciences, vol. 114, no. 3, pp. 239-247, 2010.

[42] Y. Chiba, Y. Takada, S. Miyamoto, M. Mitsui-Saito, H. Karaki, and M. Misawa, "Augmented acetylcholine-induced, Rho-mediated $\mathrm{Ca}^{2+}$ sensitization of bronchial smooth muscle contraction in antigen-induced airway hyperresponsive rats," British Journal of Pharmacology, vol. 127, no. 3, pp. 597-600, 1999.

[43] Y. Chiba, H. Sakai, H. Wachi, H. Sugitani, Y. Seyama, and M. Misawa, "Upregulation of rhoA mRNA in bronchial smooth muscle of antigen-induced airway hyperresponsive rats," Journal of Smooth Muscle Research, vol. 39, no. 6, pp. 221-228, 2003.

[44] M. F. Moffatt, I. G. Gut, F. Demenais et al., "A large-scale, consortium-based genomewide association study of asthma," The New England Journal of Medicine, vol. 363, no. 13, pp. 12111221, 2010.

[45] M. F. Moffatt, M. Kabesch, L. Liang et al., "Genetic variants regulating ORMDL3 expression contribute to the risk of childhood asthma," Nature, vol. 448, no. 7152, pp. 470-473, 2007.

[46] G. Cantero-Recasens, C. Fandos, F. Rubio-Moscardo, M. A. Valverde, and R. Vicente, "The asthma-associated ORMDL3 gene product regulates endoplasmic reticulum-mediated calcium signaling and cellular stress," Human Molecular Genetics, vol. 19, no. 1, pp. 111-121, 2009.

[47] D. E. Doherty, "The pathophysiology of airway dysfunction," American Journal of Medicine, vol. 117, supplement 12A, pp. 11S23S, 2004.

[48] P. Haldar, C. E. Brightling, B. Hargadon et al., "Mepolizumab and exacerbations of refractory eosinophilic asthma," The New England Journal of Medicine, vol. 360, no. 10, pp. 973-984, 2009.

[49] M. J. Leckie, A. Ten Brinke, J. Khan et al., "Effects of an interleukin-5 blocking monoclonal antibody on eosinophils, airway hyper-responsiveness, and the late asthmatic response," The Lancet, vol. 356, pp. 2144-2148, 2000.

[50] R. Djukanović, S. J. Wilson, M. Kraft et al., "Effects of treatment with anti-immunoglobulin E antibody omalizumab on airway inflammation in allergic asthma," American Journal of Respiratory and Critical Care Medicine, vol. 170, no. 6, pp. 583-893, 2004.

[51] G. Cox, N. C. Thomson, A. S. Rubin et al., "Asthma control during the year after bronchial thermoplasty," The New England Journal of Medicine, vol. 356, no. 13, pp. 1327-1337, 2007.

[52] J. Bousquet, E. Mantzouranis, A. A. Cruz et al., "Uniform definition of asthma severity, control, and exacerbations: document presented for the World Health Organization Consultation on Severe Asthma," Journal of Allergy and Clinical Immunology, vol. 126, pp. 926-938, 2010. 


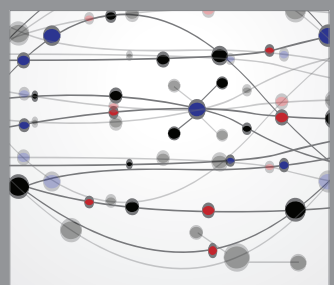

The Scientific World Journal
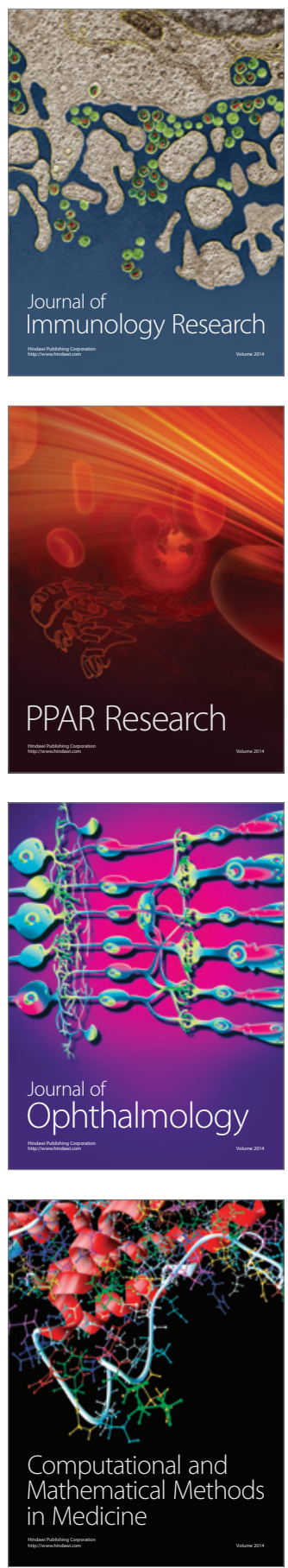

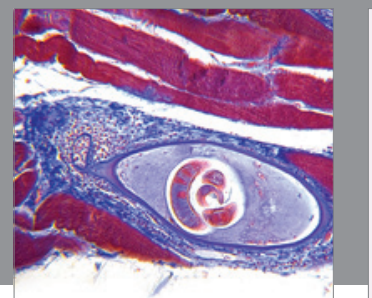

Gastroenterology

Research and Practice
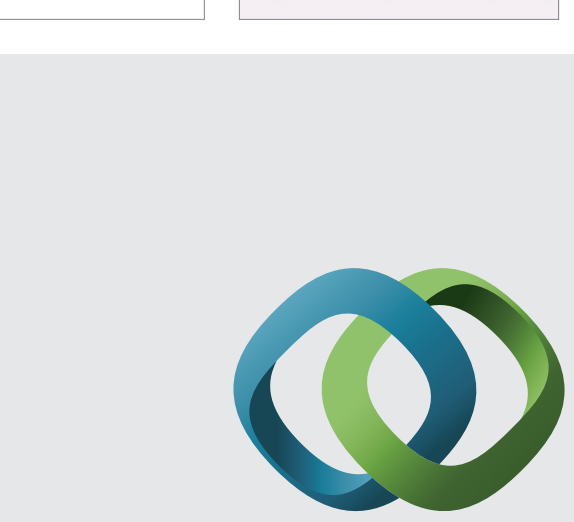

\section{Hindawi}

Submit your manuscripts at

http://www.hindawi.com
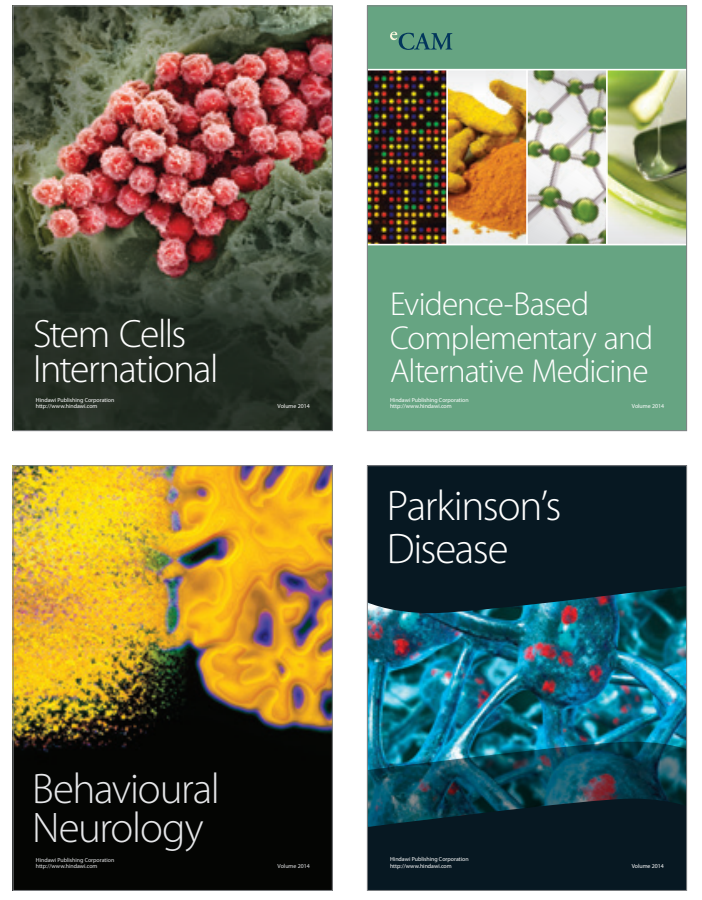
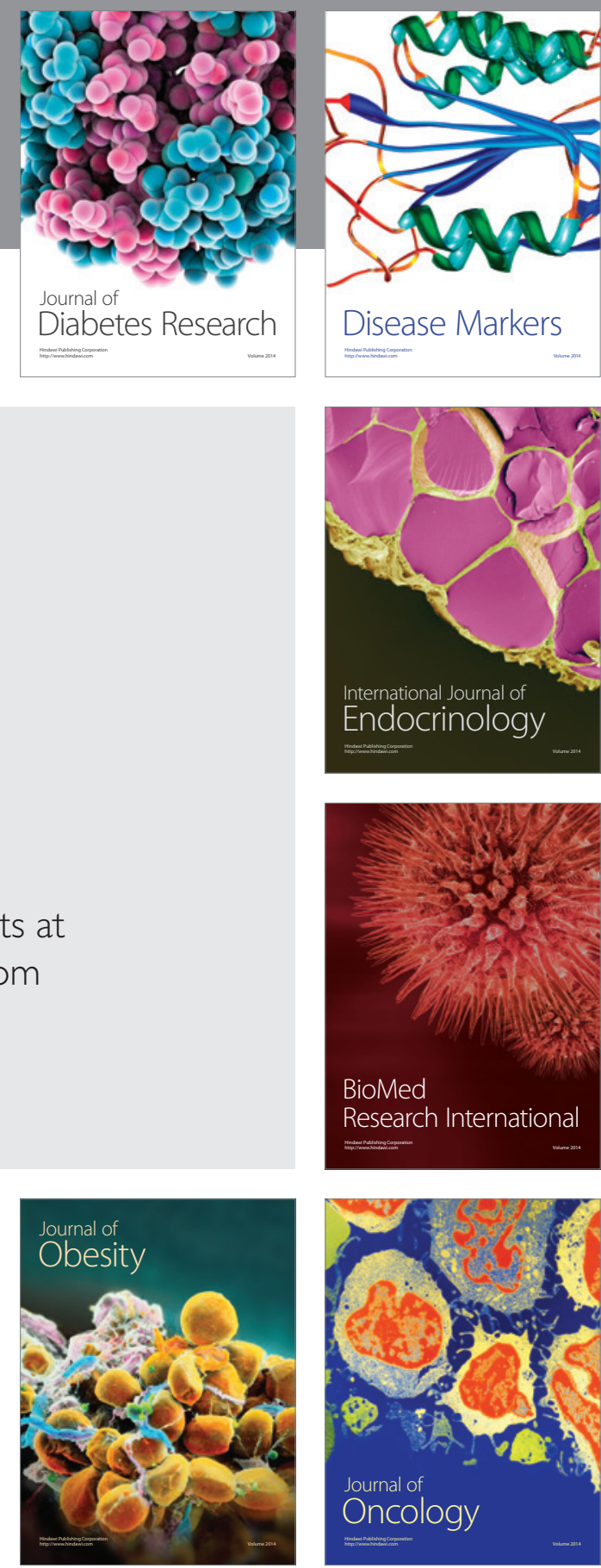

Disease Markers
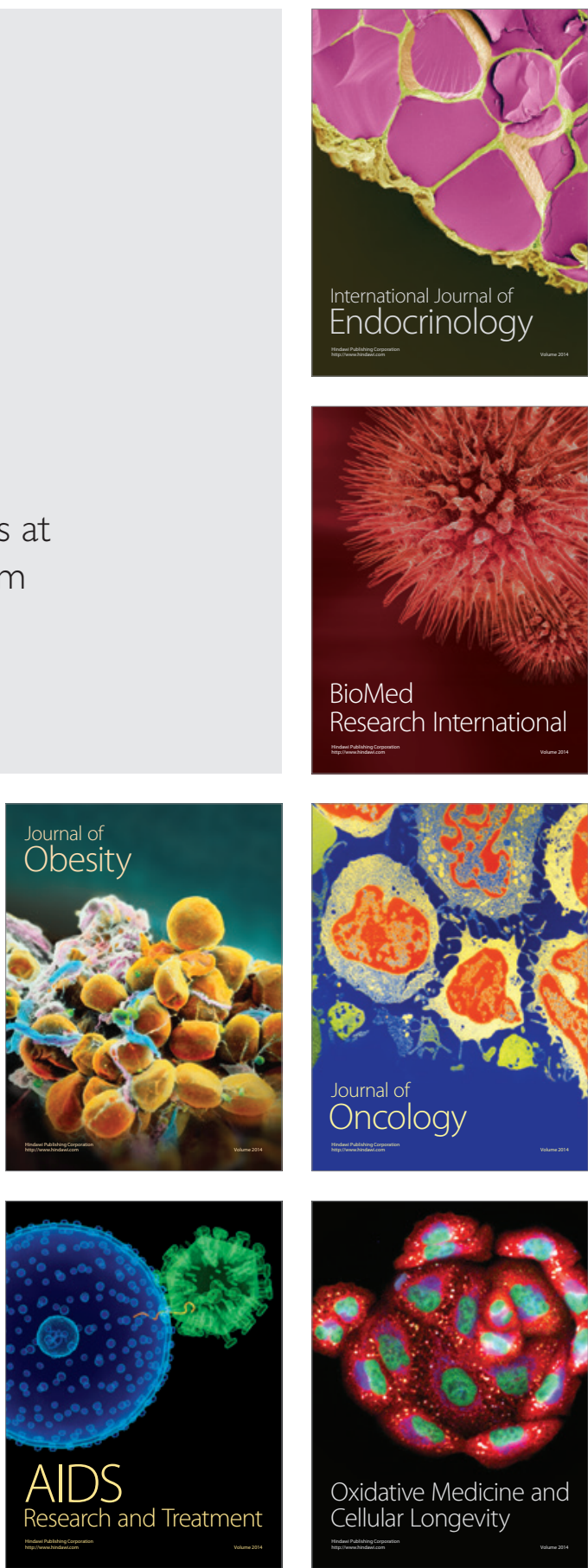